\title{
EULER SCHEME FOR SDES WITH NON-LIPSCHITZ DIFFUSION COEFFICIENT: STRONG CONVERGENCE
}

\author{
Abdel Berkaoui ${ }^{1}$, Mireille Bossy ${ }^{2}$ And Awa Diop ${ }^{2}$
}

\begin{abstract}
We consider one-dimensional stochastic differential equations in the particular case of diffusion coefficient functions of the form $|x|^{\alpha}, \alpha \in[1 / 2,1)$. In that case, we study the rate of convergence of a symmetrized version of the Euler scheme. This symmetrized version is easy to simulate on a computer. We prove its strong convergence and obtain the same rate of convergence as when the coefficients are Lipschitz.
\end{abstract}

Mathematics Subject Classification. 65C30,60H35, 65C20.

Received July 20, 2005. Revised May 30 and October 8, 2006.

\section{INTRODUCTION}

The aim of this article is to present an original technique for the strong convergence rate analysis of discretization schemes applied to SDE's with non Lipschitz diffusion coefficients. To treat the non Lipschitz property of the coefficient, we use a stochastic time change inspired from Berkaoui [2]. We restrict ourselves to one dimensional SDE's, with diffusion coefficients of the form $\sigma(x)=|x|^{\alpha}, \alpha \in[1 / 2,1)$ and we analyze the strong rate of convergence of the symmetrized Euler scheme.

More precisely, we consider $\left(X_{t}\right)_{t \geq 0}$, the $\mathbb{R}$-valued process solution to the following one-dimensional Itô stochastic differential equation

$$
X_{t}=x_{0}+\int_{0}^{t} b\left(X_{s}\right) \mathrm{d} s+\sigma \int_{0}^{t}\left|X_{s}\right|^{\alpha} \mathrm{d} W_{s}
$$

where $x_{0} \geq 0$ and $\sigma>0$ are given constants and $\left(W_{t}\right)_{t>0}$ is a one-dimensional Brownian motion defined on a given probability space $(\Omega, \mathcal{F}, \mathbb{P})$. We denote by $\left(\mathcal{F}_{t}\right)_{t \geq 0}$ the Brownian filtration. To ensure the existence of such process, we state the following hypotheses:

(H) $\alpha \in[1 / 2,1), b(0)>0$ and there exists a positive constant $K$ such that

$$
|b(x)-b(y)| \leq K|x-y|, \forall x \in \mathbb{R}, \forall y \in \mathbb{R} .
$$

\footnotetext{
Keywords and phrases. Discretization scheme, strong convergence, CIR process.

1 Dept of Statistics, University of Warwick, Gibbet Hill road, Coventry CV4 7AL, UK; berkaoui@stats.warwick.ac.uk

2 OMEGA project, INRIA Sophia Antipolis, 2004 route des Lucioles, B.P. 93, 06902 Sophia-Antipolis Cedex, France;

mireille.bossy@sophia.inria.fr and ADiop@bbspinc.com
}

(c) EDP Sciences, SMAI 2008 
Under hypotheses $(\mathrm{H})$, strong existence and uniqueness holds for the previous equation (see e.g. [10]). Moreover, as $b(x) \geq-K x$, using comparison theorem one can show that $\left(X_{t}\right)_{t \geq 0}$ is valued in $[0,+\infty)$. Then $\left(X_{t}\right)_{t \geq 0}$ is the unique strong solution to

$$
X_{t}=x_{0}+\int_{0}^{t} b\left(X_{s}\right) \mathrm{d} s+\sigma \int_{0}^{t} X_{s}^{\alpha} \mathrm{d} W_{s}
$$

Simulation schemes for (1) are motivated by applications in finance where equations like (1) model the dynamics of the short term interest rate: we refer to the Cox, Ingersoll and Ross (CIR) model [5], the Hull and White model [9] or the $S A B R$-models [8]. The solution processes are positive. This is one of the reasons to introduce these models in practice. By using the classical Euler scheme, one cannot define a positive approximation process.

We study a symmetrized Euler scheme for (1), which preserves the sign of the solution and which can be simulated easily. The symmetrized Euler scheme, introduced to treat reflected stochastic differential equations, leads to a weak convergence rate of order one for Lipschitz coefficients (see [4]). For Equation (1), the convergence in the weak sense of the present scheme has already been studied by Bossy and Diop in [3],

In this present work, we aim to study the $L^{p}$-convergence rate, which required original arguments to face the fact that the diffusion coefficient is not globally Lipschitz.

In Section 2, we describe the time discretization scheme for $\left(X_{t}\right)_{0 \leq t \leq T}$. In this particular case, symmetrized Euler scheme means that we take the absolute value of the classical Euler scheme. In Section 3, we prove the convergence. When the coefficients are globally Lipschitz, results on the convergence in $L^{p}(\Omega)$-norm for the Euler scheme are well known. A classical technique to obtain the convergence rate consists in applying the Itô formula to the function $x^{2 p}$ and the error approximation process $\mathcal{E}_{t}=\left|X_{t}-\bar{X}_{t}\right|$ and using the Lipschitz property of the coefficients (see [7]).

Here, we replace the Itô formula by the Itô-Tanaka formula, in order to deal with the absolute value of the symmetrized Euler scheme. When $1 / 2<\alpha<1$, we show that the rate of convergence is $\mathcal{O}(\sqrt{\Delta t})$. We prove the same when $\alpha=1 / 2$ with a stronger hypothesis on the drift coefficient. Let us mention two close works. First, Deelstra and Delbaen in [6] give a strong convergence result, using the Yamada's method for a quite similar scheme. Second in [1], Alfonsi analyses the (strong and weak) rate of convergence of some implicit schemes (which admit analytical solutions) in the special case of the CIR process and compare them numerically with the Deelstra and Delbaen scheme's and with the present one.

\section{ON THE SYMMETRIZED EulER SCHEME FOR (1)}

In all what follows, we assume hypotheses $(\mathrm{H})$ even if this is no more explicitly mentioned.

\subsection{The scheme and notations} by

Let $\left(X_{t}\right)_{t \geq 0}$ be given by (1). For a fixed time $T>0$, we define a discretization scheme $\left(\bar{X}_{t_{k}}, k=0, \ldots, N\right)$

$$
\left\{\begin{array}{l}
\bar{X}_{0}=x_{0}>0 \\
\bar{X}_{t_{k+1}}=\left|\bar{X}_{t_{k}}+b\left(\bar{X}_{t_{k}}\right) \Delta t+\sigma \bar{X}_{t_{k}}^{\alpha}\left(W_{t_{k+1}}-W_{t_{k}}\right)\right|, k=0, \ldots, N-1,
\end{array}\right.
$$

where $N$ denotes the number of discretization times $t_{k}=k \Delta t$ and $\Delta t>0$ is a constant time step such that $N \Delta t=T$.

In the following, we use the time continuous version $\left(\bar{X}_{t}\right)_{0 \leq t \leq T}$ :

$$
\bar{X}_{t}=\left|\bar{X}_{\eta(t)}+(t-\eta(t)) b\left(\bar{X}_{\eta(t)}\right)+\sigma \bar{X}_{\eta(t)}^{\alpha}\left(W_{t}-W_{\eta(t)}\right)\right|,
$$


where $\eta(s)=\sup _{k \in\{1, \ldots, N\}}\left\{t_{k} ; t_{k} \leq s\right\}$. The process $\left(\bar{X}_{t}\right)_{0 \leq t \leq T}$ takes positive values. By using the Tanaka's formula, we can easily show, by induction on each subinterval $\left[t_{k}, t_{k+1}\right)$ for $k=0$ to $N-1$, that $(\bar{X})$ is a continuous semimartingale with a continuous local time $\left(L_{t}^{0}(\bar{X}), 0 \leq t \leq T\right)$ at point 0 . Indeed, for any $t \in[0, T]$, if we set

$$
\bar{Z}_{t}=\bar{X}_{\eta(t)}+b\left(\bar{X}_{\eta(t)}\right)(t-\eta(t))+\sigma \bar{X}_{\eta(t)}^{\alpha}\left(W_{t}-W_{\eta(t)}\right)
$$

then, $\bar{X}_{t}=\left|\bar{Z}_{t}\right|$ and

$$
\bar{X}_{t}=x_{0}+\int_{0}^{t} \operatorname{sgn}\left(\bar{Z}_{s}\right) b\left(\bar{X}_{\eta(s)}\right) \mathrm{d} s+\sigma \int_{0}^{t} \operatorname{sgn}\left(\bar{Z}_{s}\right) \bar{X}_{\eta(s)}^{\alpha} \mathrm{d} W_{s}+\frac{1}{2} L_{t}^{0}(\bar{X}),
$$

where $\operatorname{sgn}(x):=1-2 \mathbb{1}_{(x \leq 0)}$.

The following lemma is a compilation of Lemmas 2.1, 3.1 and 4.1 in [3]. It ensures the existence of all positive moments of $(X)$ and $(\bar{X})$ as well as some negative moments of $(X)$ :

Lemma 2.1. For any $x_{0}>0$, for any $p \geq 1$, there exists a positive constant $C$, depending on $p$, but also on the parameters $b(0), K, \sigma, \alpha$ and $T$, such that

$$
\mathbb{E}\left(\sup _{t \in[0, T]} X_{t}^{2 p}\right)+\mathbb{E}\left(\sup _{t \in[0, T]} \bar{X}_{t}^{2 p}\right) \leq C\left(1+x_{0}^{2 p}\right)
$$

When $1 / 2<\alpha<1$, for any $p>0$,

$$
\sup _{t \in[0, T]} \mathbb{E}\left(X_{t}^{-p}\right) \leq C\left(1+x_{0}^{-p}\right)
$$

When $\alpha=\frac{1}{2}$, for any $p$ such that $1<p<\frac{2 b(0)}{\sigma^{2}}-1$

$$
\mathbb{E}\left(X_{t}^{-p}\right) \leq C x_{0}^{-p}
$$

The proof can be found in [3].

\subsection{Strong convergence}

Theorem 2.2. Consider $\left(X_{t}\right)_{0 \leq t \leq T}$, the solution of (1) and $\left(\bar{X}_{t}\right)_{0 \leq t \leq T}$, the corresponding symmetrized Euler scheme given by (2). Let $\Delta t$ satisfying $\Delta t \leq 1 /(2 K)$.

i) For all $1 / 2<\alpha<1$, for all $p \geq 1$, there exists a positive constant $C(T, p)$ depending on $\alpha, \sigma, b, T$ and $x_{0}$, increasing in $T$ and $p$, such that

$$
\left[\mathbb{E} \sup _{0 \leq t \leq T}\left|X_{t}-\bar{X}_{t}\right|^{2 p}\right]^{1 / 2 p} \leq C(T, p) \sqrt{\Delta t}
$$

ii) When $\alpha=1 / 2$, let $\Delta t$ satisfying $\Delta t \leq 1 /(2 K) \wedge x_{0}$. The estimate (9) holds if we assume also that

$$
\frac{\sigma^{2}}{8}\left(\frac{2 b(0)}{\sigma^{2}}-1\right)^{2}>\mathcal{K}(4 p)
$$

with $\mathcal{K}(p)=K(4 p-1) \vee(4 \sigma \alpha(2 p-1))^{2}$. 
We emphasize the difference between the situation $1 / 2<\alpha<1$ and $\alpha=1 / 2$. Let $\tau_{0}=\inf \left\{t \geq 0 ; X_{t}=0\right\}$. When $1 / 2<\alpha<1$ and $x_{0}>0$, Feller's test on process $(X)$ shows that it is enough to suppose $b(0)>0$ as in $(\mathrm{H})$ to ensure that $\mathbb{P}\left(\tau_{0}=\infty\right)=1$. When $\alpha=1 / 2,(X)$ satisfies the equation

$$
X_{t}=x_{0}+\int_{0}^{t} b\left(X_{s}\right) \mathrm{d} s+\sigma \int_{0}^{t} \sqrt{X_{s}} \mathrm{~d} W_{s}, \quad 0 \leq t \leq T
$$

When $b(x)$ is of the form $a-b x$, with $a>0,(X)$ is the classical CIR process. When $b(x)=a>0,(X)$ is the square of a Bessel process. When $x_{0}>0$, one can show that $\mathbb{P}\left(\tau_{0}=\infty\right)=1$ for any drift $b(x)$ satisfying $(\mathrm{H})$ and $b(0) \geq \sigma^{2} / 2$, by using the comparison theorem and Feller's test. In addition, if $b(0) \geq \sigma^{2} / 2$, we are able to control the exponential inverse moment of the CIR-like process $(X)$ (see Lem. 3.1 below). The constraint (10) on $b(0)$ and $\sigma$ is stronger than $b(0) \geq \sigma^{2} / 2$. This is due to the particular use of Lemma 3.1 below in the proof of Theorem 2.2.

\section{ProOF}

In all the sequel, $C$ denotes a positive constant depending on $p$ or on the parameters $b, \sigma, \alpha$ and $x_{0}$ of the model but not on $\Delta t$. We also use the following notation

$$
\mathcal{O}_{\exp }(\Delta t)=\exp \left(-\frac{C}{\Delta t^{\beta}}\right), \text { for some } \beta>0
$$

\subsection{Preliminaries}

In this subsection, we gather a few results on exponential moments of $(X)$ and on the behavior of the approximation processes $(\bar{X})$ and $(\bar{Z})$ visiting zero. Those results are crucial in the proof of convergence.

Lemma 3.1. Let $\left(X_{t}\right)_{0 \leq t \leq T}$ be the solution of (1) with $1 / 2<\alpha<1$. For all $\mu \geq 0$, there exists a positive constant $C(T, \mu)$, increasing in $\mu$ and $T$, depending also on $b \sigma, \alpha$ and $x_{0}$ such that

$$
\mathbb{E} \exp \left(\mu \int_{0}^{T} \frac{\mathrm{d} s}{X_{s}^{2(1-\alpha)}}\right) \leq C(T, \mu)
$$

When $\alpha=1 / 2$, the inequality (12) holds if $\mu \leq \frac{\nu^{2} \sigma^{2}}{8}$ and $\nu=\left(\frac{2 b(0)}{\sigma^{2}}-1\right) \geq 0$.

The proof can be found in [3].

Lemma 3.2. Let $\left(\bar{X}_{t}\right)_{0 \leq t \leq T}$ be the approximation process defined by (3) and $\left(\bar{Z}_{t}\right)_{0 \leq t \leq T}$ given by (4). Let $\Delta t \leq 1 /(2 K)$.

i) If $1 / 2<\alpha<1$, then $\sup _{t \in[0, T]} \mathbb{P}\left(\bar{Z}_{t} \leq 0\right) \leq \mathcal{O}_{\exp }(\Delta t)$.

ii) If $\alpha=1 / 2$ and $\Delta t \leq 1 /(2 K) \wedge x_{0}, \sup _{t \in[0, T]} \mathbb{P}\left(\bar{Z}_{t} \leq 0\right) \leq C\left(\frac{\Delta t}{x_{0}}\right)^{\frac{b(0)}{\sigma^{2}}}$. 
Proof. Under (H), for any $x \geq 0, b(x) \geq b(0)-K x$. Thus, by (4),

$$
\begin{aligned}
\mathbb{P}\left(\bar{Z}_{t} \leq 0\right) & \leq \mathbb{P}\left(\bar{X}_{\eta(t)}+\left(b(0)-K \bar{X}_{\eta(t)}\right)(t-\eta(t))+\sigma \bar{X}_{\eta(t)}^{\alpha}\left(W_{t}-W_{\eta(t)}\right) \leq 0\right) \\
& =\mathbb{P}\left(W_{t}-W_{\eta(t)} \leq \frac{-\bar{X}_{\eta(t)}(1-K(t-\eta(t)))-b(0)(t-\eta(t))}{\sigma \bar{X}_{\eta(t)}^{\alpha}}, \bar{X}_{\eta(t)}>0\right) \\
& \leq \frac{1}{2} \mathbb{E}\left[\exp \left(-\frac{\left(\bar{X}_{\eta(t)}(1-K(t-\eta(t)))+b(0)(t-\eta(t))\right)^{2}}{2 \sigma^{2}(t-\eta(t)) \bar{X}_{\eta(t)}^{2 \alpha}}\right) \mathbb{1}_{\left\{\bar{X}_{\eta(t)}>0\right\}}\right] .
\end{aligned}
$$

When $1 / 2<\alpha<1$, noting that $(1-K \Delta t) \geq \frac{1}{2}$, we have

$$
\mathbb{P}\left(\bar{Z}_{t} \leq 0\right) \leq \frac{1}{2} \mathbb{E}\left[\exp \left(-\frac{\bar{X}_{\eta(t)}^{2(1-\alpha)}}{8 \sigma^{2} \Delta t}\right) \exp \left(-\frac{b(0)}{2 \sigma^{2} \bar{X}_{\eta(t)}^{2 \alpha-1}}\right) \mathbb{1}_{\left\{\bar{X}_{\eta(t)}>0\right\}}\right]
$$

We distinguish the events $\left(\bar{X}_{\eta(t)} \leq \sqrt{\Delta t}\right)$ and $\left(\bar{X}_{\eta(t)}>\sqrt{\Delta t}\right)$ and easily conclude that $\mathbb{P}\left(\bar{Z}_{t} \leq 0\right)=\mathcal{O}_{\exp }(\Delta t)$.

When $\alpha=1 / 2$, we deduce from the previous computation that

$$
\mathbb{P}\left(\bar{Z}_{t} \leq 0 / \bar{X}_{\eta(t)}\right) \leq \frac{1}{2} \exp \left(-\frac{\bar{X}_{\eta(t)}}{8 \sigma^{2} \Delta t}\right) .
$$

The Lemma 3.6 in [3] ensures that, for any $\gamma \geq 1$,

$$
\sup _{k \leq N} \mathbb{E} \exp \left(-\frac{\bar{X}_{t_{k}}}{\gamma \sigma^{2} \Delta t}\right) \leq C\left(\frac{\Delta t}{x_{0}}\right)^{\frac{2 b(0)}{\sigma^{2}}\left(1-\frac{1}{2 \gamma}\right)},
$$

which ends the proof.

\subsection{Proof of Theorem 2.2}

We start with estimating the local error of the scheme (3):

Lemma 3.3. For all $p \geq 1$ and all $1 / 2 \leq \alpha<1$, there exists a positive constant $C$, depending on $b(0), \sigma, \alpha, p$, $K$ and $T$ but not on $\Delta t$ such that

$$
\sup _{t \in[0, T]} \mathbb{E}\left\{\left|\bar{X}_{t}-\bar{X}_{\eta(t)}\right|^{2 p}\right\} \leq C \Delta t^{p}
$$

Proof. Indeed,

$$
\sup _{t \in[0, T]} \mathbb{E}\left|\bar{X}_{t}-\bar{X}_{\eta(t)}\right|^{2 p} \leq \sup _{k=0 \ldots N-1} \sup _{t \in\left[t_{k}, t_{k+1}\right]} \mathbb{E}\left|\bar{X}_{t}-\bar{X}_{t_{k}}\right|^{2 p}
$$

By using (3), we have for $t \in\left[t_{k}, t_{k+1}\right]$,

$$
\left|\bar{X}_{t}-\bar{X}_{t_{k}}\right|^{2 p} \leq\left|b\left(\bar{X}_{t_{k}}\right)\left(t-t_{k}\right)+\sigma \bar{X}_{t_{k}}^{\alpha}\left(W_{t}-W_{t_{k}}\right)\right|^{2 p} \leq 2^{2 p-1} \Delta t^{2 p}\left|b\left(\bar{X}_{t_{k}}\right)\right|^{2 p}+2^{2 p-1} \sigma^{2 p} \bar{X}_{t_{k}}^{2 p \alpha}\left|W_{t}-W_{t_{k}}\right|^{2 p}
$$


But $\left|b\left(\bar{X}_{t_{k}}\right)\right|^{2 p} \leq 2^{2 p-1}\left(b(0)^{2 p}+K^{2 p}\left|\bar{X}_{t_{k}}\right|^{2 p}\right)$ and we easily conclude by using Lemma 2.1 that

$$
\begin{aligned}
\sup _{t \in\left[t_{k}, t_{k+1}\right]} \mathbb{E}\left|\bar{X}_{t}-\bar{X}_{t_{k}}\right|^{2 p} & \leq C 2^{2 p-1} \Delta t^{2 p}+C 2^{2 p-1} \sigma^{2 p} \sup _{t \in\left[t_{k}, t_{k+1}\right]}\left(\mathbb{E}\left|W_{t}-W_{t_{k}}\right|^{4 p}\right)^{1 / 2} \\
& \leq C(p) \Delta t^{p} .
\end{aligned}
$$

We define the process $(\gamma(t))_{t \geq 0}$ by

$$
\gamma(t)=\int_{0}^{t} \frac{\mathrm{d} s}{\left(X_{s}^{1-\alpha}+\bar{X}_{\eta(s)}^{1-\alpha}\right)^{2}} .
$$

Note that $\gamma(t)$ is well defined. Indeed, almost surely the process $(\bar{X})$ is positive and under $(\mathrm{H})$ (and the condition $b(0)>\sigma^{2} / 2$, when $\left.\alpha=1 / 2\right)$, the process $(X)$ is almost surely strictly positive.

Let $\tau_{\lambda}$ be the stopping time defined by

$$
\tau_{\lambda}=\inf \{s \in[0, T], \quad \gamma(s)+s \geq \lambda\}
$$

with $\inf \emptyset=T$. We begin the convergence analysis, considering the strong error at the stopping time $\tau_{\lambda}$ :

Lemma 3.4. For all $\lambda \geq 0$ and all integer $p \geq 1$, there exists a positive constant $C(p)$ depending on $b(0), \sigma$, $\alpha, p, K$ and $T$ but not on $\Delta t$ and $\lambda$, such that

$$
\mathbb{E}\left|\bar{X}_{\tau_{\lambda}}-X_{\tau_{\lambda}}\right|^{2 p} \leq \exp (\mathcal{K}(p) \lambda) C(p) \Delta t^{p}
$$

with $\mathcal{K}(p)=K(4 p-1) \vee(4 \sigma \alpha(2 p-1))^{2}$.

Proof. The error process $\left(\mathcal{E}_{t}\right)_{0 \leq t \leq T}$ defined by $\mathcal{E}_{t}:=\bar{X}_{t}-X_{t}$, satisfies

$$
\mathcal{E}_{t}=\int_{0}^{t}\left(b\left(\bar{X}_{\eta(s)}\right) \operatorname{sgn}\left(\bar{Z}_{s}\right)-b\left(X_{s}\right)\right) \mathrm{d} s+\sigma \int_{0}^{t}\left(\bar{X}_{\eta(s)}^{\alpha} \operatorname{sgn}\left(\bar{Z}_{s}\right)-X_{s}^{\alpha}\right) \mathrm{d} W_{s}+\frac{1}{2} L_{t}^{0}(\bar{X}) .
$$

For an arbitrary stopping time $\tau$ valued in $[0, T]$, we apply the Itô formula to $\mathcal{E}_{t}^{2 p}$, between 0 and $\tau$. As $\int_{0}^{\tau}\left(\mathcal{E}_{s}\right)^{2 p-1} \mathrm{~d} L_{s}^{0}(\bar{X})=\int_{0}^{\tau}\left(-X_{s}\right)^{2 p-1} \mathrm{~d} L_{s}^{0}(\bar{X}) \leq 0$, we obtain

$$
\begin{aligned}
\mathbb{E}\left|\mathcal{E}_{\tau}\right|^{2 p} \leq & 2 p \mathbb{E}\left[\int_{0}^{\tau}\left(\mathcal{E}_{s}\right)^{2 p-1}\left(b\left(\bar{X}_{\eta(s)}\right) \operatorname{sgn}\left(\bar{Z}_{s}\right)-b\left(X_{s}\right)\right) \mathrm{d} s\right] \\
& +p(2 p-1) \sigma^{2} \mathbb{E}\left[\int_{0}^{\tau}\left(\mathcal{E}_{s}\right)^{2 p-2}\left(\bar{X}_{\eta(s)}^{\alpha} \operatorname{sgn}\left(\bar{Z}_{s}\right)-X_{s}^{\alpha}\right)^{2} \mathrm{~d} s\right] \\
\leq & 2 p \mathbb{E}\left[\int_{0}^{\tau}\left(\mathcal{E}_{s}\right)^{2 p-1}\left(b\left(\bar{X}_{\eta(s)}\right)-b\left(X_{s}\right)\right) \mathrm{d} s\right] \\
& +2 p(2 p-1) \sigma^{2} \mathbb{E}\left[\int_{0}^{\tau}\left(\mathcal{E}_{s}\right)^{2 p-2}\left(\bar{X}_{\eta(s)}^{\alpha}-X_{s}^{\alpha}\right)^{2} \mathrm{~d} s\right] \\
& +2 \mathbb{E}\left[\int_{0}^{\tau}\left\{2 p\left|\mathcal{E}_{s}\right|^{2 p-1}\left|b\left(\bar{X}_{\eta(s)}\right)\right|+2 p(2 p-1) \sigma^{2}\left(\mathcal{E}_{s}\right)^{2 p-2} \bar{X}_{\eta(s)}^{2 \alpha}\right\} \mathbb{1}_{\left\{\bar{Z}_{s} \leq 0\right\}} \mathrm{d} s\right] .
\end{aligned}
$$

Thanks to Lemma 2.1 , we easily check that for any $\beta \geq 0$,

$$
\mathbb{E}\left[\int_{0}^{\tau}\left|\mathcal{E}_{s}\right|^{\beta}\left(\left|b\left(\bar{X}_{\eta(s)}\right)\right|+\bar{X}_{\eta(s)}^{2 \alpha}\right) \mathbb{1}_{\left\{\bar{Z}_{s} \leq 0\right\}} \mathrm{d} s\right] \leq C \sqrt{\sup _{t \in[0, T]} \mathbb{P}\left(\bar{Z}_{t} \leq 0\right)} .
$$


When $1 / 2<\alpha<1$, by Lemma 3.2, we conclude that

$$
\begin{aligned}
\mathbb{E}\left|\mathcal{E}_{\tau}\right|^{2 p} \leq & 2 p \mathbb{E}\left[\int_{0}^{\tau}\left(\mathcal{E}_{s}\right)^{2 p-1}\left(b\left(\bar{X}_{\eta(s)}\right)-b\left(X_{s}\right)\right) \mathrm{d} s\right] \\
& +2 p(2 p-1) \sigma^{2} \mathbb{E}\left[\int_{0}^{\tau}\left(\mathcal{E}_{s}\right)^{2 p-2}\left(\bar{X}_{\eta(s)}^{\alpha}-X_{s}^{\alpha}\right)^{2} \mathrm{~d} s\right]+\mathcal{O}_{\exp }(\Delta t) .
\end{aligned}
$$

When $\alpha=1 / 2$, in the case of CIR-like processes, from Lemma 3.2 we only have

$$
\sqrt{\sup _{t \in[0, T]} \mathbb{P}\left(\bar{Z}_{t} \leq 0\right)} \leq C \Delta t^{\frac{b(0)}{2 \sigma^{2}}} .
$$

When we sum up the case $\alpha=1 / 2$ and $1 / 2<\alpha<1$, (17) becomes

$$
\begin{aligned}
\mathbb{E}\left|\mathcal{E}_{\tau}\right|^{2 p} \leq & 2 p \mathbb{E}\left[\int_{0}^{\tau}\left(\mathcal{E}_{s}\right)^{2 p-1}\left(b\left(\bar{X}_{\eta(s)}\right)-b\left(X_{s}\right)\right) \mathrm{d} s\right] \\
& +2 p(2 p-1) \sigma^{2} \mathbb{E}\left[\int_{0}^{\tau}\left(\mathcal{E}_{s}\right)^{2 p-2}\left(\bar{X}_{\eta(s)}^{\alpha}-X_{s}^{\alpha}\right)^{2} \mathrm{~d} s\right]+C \Delta t^{\frac{b(0)}{2 \sigma^{2}} \wedge p}
\end{aligned}
$$

In the case $\alpha=1 / 2$, the convergence rate seems to decrease from $\Delta t^{p}$ to $\Delta t^{\frac{b(0)}{2 \sigma^{2}} \wedge p}$. This also occurs when one analyzes the weak convergence rate in [3]. Indeed, for smooth enough test function $f(x)$, one has that $\left|\mathbb{E} f\left(X_{T}\right)-\mathbb{E} f\left(\bar{X}_{T}\right)\right| \leq C \Delta t^{\frac{b(0)}{\sigma^{2}} \wedge 1}$ instead of $C \Delta t$, and such behavior is confirmed by numerical experiments (see $[1])$.

Here, due to constraint (10), we are in the situation where $\frac{b(0)}{2 \sigma^{2}} \geq p$ and the convergence rate does not change when $\alpha=1 / 2$. The reason to impose this so strong condition (10) will appear later, when we will try to apply Lemma 3.1 in the case $\alpha=1 / 2$, in the proof of Theorem 2.2.

In view of (13), we have

$$
\mathbb{E}\left[\int_{0}^{\tau}\left(\mathcal{E}_{s}\right)^{2 p-2}\left(\bar{X}_{\eta(s)}^{\alpha}-X_{s}^{\alpha}\right)^{2} \mathrm{~d} s\right]=\mathbb{E}\left[\int_{0}^{\tau}\left(\mathcal{E}_{s}\right)^{2 p-2}\left(\bar{X}_{\eta(s)}^{\alpha}-X_{s}^{\alpha}\right)^{2}\left(X_{s}^{1-\alpha}+\bar{X}_{\eta(s)}^{1-\alpha}\right)^{2} \mathrm{~d} \gamma(s)\right] .
$$

We remark that, for all $1 / 2 \leq \alpha \leq 1$,

$$
\forall x \geq 0, y \geq 0, \quad\left|x^{\alpha}-y^{\alpha}\right|\left(x^{1-\alpha}+y^{1-\alpha}\right) \leq 2 \alpha|x-y| .
$$

We deduce from (19) that

$$
\mathbb{E} \int_{0}^{\tau}\left(\mathcal{E}_{s}\right)^{2 p-2}\left(\bar{X}_{\eta(s)}^{\alpha}-X_{s}^{\alpha}\right)^{2} \mathrm{~d} s \leq 4 \alpha^{2} \mathbb{E} \int_{0}^{\tau}\left(\mathcal{E}_{s}\right)^{2(p-1)}\left(\bar{X}_{\eta(s)}-X_{s}\right)^{2} \mathrm{~d} \gamma(s),
$$

and then (18) becomes

$$
\begin{aligned}
\mathbb{E}\left|\mathcal{E}_{\tau}\right|^{2 p} \leq & 2 p K \mathbb{E}\left[\int_{0}^{\tau}\left|\mathcal{E}_{s}\right|^{2 p-1}\left|\bar{X}_{\eta(s)}-X_{s}\right| \mathrm{d} s\right] \\
& +2 p(2 p-1) \sigma^{2} 4 \alpha^{2} \mathbb{E}\left[\int_{0}^{\tau}\left(\mathcal{E}_{s}\right)^{2(p-1)}\left(\bar{X}_{\eta(s)}-X_{s}\right)^{2} \mathrm{~d} \gamma(s)\right]+C \Delta t^{p} .
\end{aligned}
$$

Now, we remark that for $r=1,2$,

$$
\left|\mathcal{E}_{s}\right|^{2 p-r}\left|\bar{X}_{\eta(s)}-X_{s}\right|^{r} \leq\left|\mathcal{E}_{s}\right|^{2 p-r}\left(\left|\mathcal{E}_{s}\right|+\left|\bar{X}_{\eta(s)}-\bar{X}_{s}\right|\right)^{r} \leq r\left|\mathcal{E}_{s}\right|^{2 p-r}\left(\left|\mathcal{E}_{s}\right|^{r}+\left|\bar{X}_{\eta(s)}-\bar{X}_{s}\right|^{r}\right) .
$$


Moreover, by the Young inequality we have

$$
\left|\mathcal{E}_{s}\right|^{2 p-r}\left|\bar{X}_{\eta(s)}-\bar{X}_{s}\right|^{r} \leq \frac{2 p-r}{2 p}\left(\mathcal{E}_{s}\right)^{2 p}+\frac{r}{2 p}\left|\bar{X}_{\eta(s)}-\bar{X}_{s}\right|^{2 p}
$$

so that, for $r=1,2$,

$$
\left|\mathcal{E}_{s}\right|^{2 p-r}\left|\bar{X}_{\eta(s)}-X_{s}\right|^{r} \leq r\left(1+\frac{2 p-r}{2 p}\right)\left(\mathcal{E}_{s}\right)^{2 p}+\frac{r^{2}}{2 p}\left|\bar{X}_{\eta(s)}-\bar{X}_{s}\right|^{2 p}
$$

By using (21) with $r=1$ and $r=2$ in (20), we obtain

$$
\begin{aligned}
\mathbb{E}\left|\mathcal{E}_{\tau}\right|^{2 p} \leq & \mathcal{K}(p) \mathbb{E}\left[\int_{0}^{\tau}\left(\mathcal{E}_{s}\right)^{2 p} \mathrm{~d}(s+\gamma(s))\right] \\
& +\left[K \vee(2 p-1)(4 \alpha \sigma)^{2}\right] \mathbb{E}\left[\int_{0}^{\tau}\left|\bar{X}_{\eta(s)}-\bar{X}_{s}\right|^{2 p} \mathrm{~d}(s+\gamma(s))\right]+C \Delta t^{p},
\end{aligned}
$$

with $\mathcal{K}(p)=K(4 p-1) \vee(4 \sigma \alpha(2 p-1))^{2}$. Furthermore, we observe that

$$
\mathbb{E}\left[\int_{0}^{\tau}\left|\bar{X}_{\eta(s)}-\bar{X}_{s}\right|^{2 p} \mathrm{~d}(s+\gamma(s))\right] \leq \mathbb{E}\left[\int_{0}^{T}\left|\bar{X}_{\eta(s)}-\bar{X}_{s}\right|^{2 p}\left(1+\frac{1}{X_{s}^{2-2 \alpha}}\right) \mathrm{d} s\right] .
$$

By using the local error estimate in Lemma 3.3 , for any couple $(a, b)$ such that $1 / a+1 / b=1$, we have

$$
\begin{aligned}
\mathbb{E}\left[\int_{0}^{\tau}\left|\bar{X}_{\eta(s)}-\bar{X}_{s}\right|^{2 p} \mathrm{~d}(s+\gamma(s))\right] & \leq C \Delta t^{p}+\int_{0}^{T}\left(\mathbb{E}\left|\bar{X}_{\eta(s)}-\bar{X}_{s}\right|^{2 p b}\right)^{\frac{1}{b}}\left(\mathbb{E}\left(\frac{1}{X_{s}^{a(2-2 \alpha)}}\right)\right)^{\frac{1}{a}} \mathrm{~d} s \\
& \leq C \Delta t^{p}\left(1+\sup _{t \in[0, T]}\left(\mathbb{E}\left(\frac{1}{X_{t}^{a(2-2 \alpha)}}\right)\right)^{\frac{1}{a}}\right) .
\end{aligned}
$$

We apply Lemma 2.1 to upper-bound the negative moment of $(X)$. By condition (10), as $\frac{2 b(0)}{\sigma^{2}}-1>2$, we can choose $a=2$, even if $\alpha=1 / 2$. Then

$$
\mathbb{E}\left|\mathcal{E}_{\tau}\right|^{2 p} \leq \mathcal{K}(p) \mathbb{E}\left[\int_{0}^{\tau}\left(\mathcal{E}_{s}\right)^{2 p} \mathrm{~d}(s+\gamma(s))\right]+C \Delta t^{p} .
$$

Now, we choose $\tau=\tau_{\lambda}$ defined in (14). Noting that $\tau_{\lambda}+\gamma\left(\tau_{\lambda}\right)=\lambda$, we apply the change of time $u=s+\gamma(s)$ in the integral above:

$$
\mathbb{E}\left|\mathcal{E}_{\tau_{\lambda}}\right|^{2 p} \leq \mathcal{K}(p)\left[\int_{0}^{\lambda} \mathbb{E}\left|\mathcal{E}_{\tau_{u}}\right|^{2 p} \mathrm{~d} u\right]+C \Delta t^{p} .
$$

By Gronwall's lemma, we conclude that

$$
\mathbb{E}\left|\mathcal{E}_{\tau_{\lambda}}\right|^{2 p} \leq C \Delta t^{p} \exp (\mathcal{K}(p) \lambda)
$$


Proof of Theorem 2.2. We proceed as before to get for all $t \in[0, T]$,

$$
\begin{aligned}
\left(\mathcal{E}_{t}\right)^{2 p} \leq & 2 p \int_{0}^{t}\left(\mathcal{E}_{s}\right)^{2 p-1}\left(b\left(\bar{X}_{\eta(s)}\right) \operatorname{sgn}\left(\bar{Z}_{s}\right)-b\left(X_{s}\right)\right) \mathrm{d} s \\
& +p(2 p-1) \sigma^{2} \int_{0}^{t}\left(\mathcal{E}_{s}\right)^{2 p-2}\left(\bar{X}_{\eta(s)}^{\alpha} \operatorname{sgn}\left(\bar{Z}_{s}\right)-X_{s}^{\alpha}\right)^{2} \mathrm{~d} s \\
& +2 p \sigma \int_{0}^{t}\left(\mathcal{E}_{s}\right)^{2 p-1}\left(\bar{X}_{\eta(s)}^{\alpha} \operatorname{sgn}\left(\bar{Z}_{s}\right)-X_{s}^{\alpha}\right) \mathrm{d} W_{s}
\end{aligned}
$$

and by the Burkholder-Davis-Gundy inequality,

$$
\begin{aligned}
\mathbb{E} \sup _{0 \leq s \leq T}\left|\mathcal{E}_{t}\right|^{2 p} \leq & C \mathbb{E}\left[\int_{0}^{T}\left|\mathcal{E}_{s}\right|^{2 p-1}\left|b\left(\bar{X}_{\eta(s)}\right) \operatorname{sgn}\left(\bar{Z}_{s}\right)-b\left(X_{s}\right)\right| \mathrm{d} s\right] \\
& +C \mathbb{E}\left[\int_{0}^{T}\left(\mathcal{E}_{s}\right)^{2 p-2}\left(\bar{X}_{\eta(s)}^{\alpha} \operatorname{sgn}\left(\bar{Z}_{s}\right)-X_{s}^{\alpha}\right)^{2} \mathrm{~d} s\right] \\
& +C \sqrt{\mathbb{E} \int_{0}^{T}\left(\mathcal{E}_{s}\right)^{4 p-2}\left(\bar{X}_{\eta(s)}^{\alpha} \operatorname{sgn}\left(\bar{Z}_{s}\right)-X_{s}^{\alpha}\right)^{2} \mathrm{~d} s .}
\end{aligned}
$$

Again we use (16) and Lemma 3.2, (together with the condition (10) when $\alpha=1 / 2$ ) to get

$$
\begin{aligned}
\mathbb{E} \sup _{0 \leq s \leq T}\left|\mathcal{E}_{t}\right|^{2 p} & \leq C \mathbb{E}\left[\int_{0}^{T}\left|\mathcal{E}_{s}\right|^{2 p-1}\left|\bar{X}_{\eta(s)}-X_{s}\right| \mathrm{d} s\right] \\
& +C \mathbb{E}\left[\int_{0}^{T}\left(\mathcal{E}_{s}\right)^{2 p-2}\left(\bar{X}_{\eta(s)}^{\alpha}-X_{s}^{\alpha}\right)^{2} \mathrm{~d} s\right] \\
& +C \sqrt{\mathbb{E} \int_{0}^{T}\left(\mathcal{E}_{s}\right)^{4 p-2}\left(\bar{X}_{\eta(s)}^{\alpha}-X_{s}^{\alpha}\right)^{2} \mathrm{~d} s}+C \Delta t^{p}
\end{aligned}
$$

Next, we use (19) and, by definition of $\gamma_{t}$ in (13), we have

$$
\begin{aligned}
\mathbb{E} \sup _{0 \leq s \leq T}\left|\mathcal{E}_{t}\right|^{2 p} \leq & C \mathbb{E}\left[\int_{0}^{T}\left|\mathcal{E}_{s}\right|^{2 p-1}\left|\bar{X}_{\eta(s)}-X_{s}\right| \mathrm{d} s\right] \\
& +C \mathbb{E}\left[\int_{0}^{T}\left(\mathcal{E}_{s}\right)^{2 p-2}\left(\bar{X}_{\eta(s)}-X_{s}\right)^{2} \mathrm{~d} \gamma(s)\right] \\
& +C \sqrt{\mathbb{E} \int_{0}^{T}\left(\mathcal{E}_{s}\right)^{4 p-2}\left(\bar{X}_{\eta(s)}-X_{s}\right)^{2} \mathrm{~d} \gamma(s)}+C \Delta t^{p}
\end{aligned}
$$


We use the upper bound (21) in each of the three terms above:

$$
\begin{aligned}
\mathbb{E} \sup _{0 \leq s \leq T}\left|\mathcal{E}_{t}\right|^{2 p} \leq & C \mathbb{E}\left[\int_{0}^{T}\left(\mathcal{E}_{s}\right)^{2 p} \mathrm{~d}(\gamma(s)+s)\right] \\
& +C \mathbb{E}\left[\int_{0}^{T}\left|\bar{X}_{\eta(s)}-\bar{X}_{s}\right|^{2 p} \mathrm{~d}(\gamma(s)+s)\right] \\
& +C \sqrt{\mathbb{E} \int_{0}^{T}\left(\mathcal{E}_{s}\right)^{4 p} \mathrm{~d} \gamma(s)} \\
& +C \sqrt{\mathbb{E} \int_{0}^{T}\left|\bar{X}_{\eta(s)}-\bar{X}_{s}\right|^{4 p} \mathrm{~d} \gamma(s)}+C \Delta t^{p} .
\end{aligned}
$$

As previously, $\mathbb{E}\left[\int_{0}^{T}\left|\bar{X}_{\eta(s)}-\bar{X}_{s}\right|^{2 p} \mathrm{~d}(\gamma(s)+s)\right] \leq C \Delta t^{p}$ and the same treatment on $\sqrt{\mathbb{E} \int_{0}^{T}\left|\bar{X}_{\eta(s)}-\bar{X}_{s}\right|^{4 p} \mathrm{~d} \gamma(s)}$ leads to

$$
\mathbb{E} \sup _{0 \leq s \leq T}\left|\mathcal{E}_{t}\right|^{2 p} \leq C \mathbb{E}\left[\int_{0}^{T}\left(\mathcal{E}_{s}\right)^{2 p} \mathrm{~d}(\gamma(s)+s)\right]+C \sqrt{\mathbb{E} \int_{0}^{T}\left(\mathcal{E}_{s}\right)^{4 p} \mathrm{~d} \gamma(s)}+C \Delta t^{p} .
$$

Again, we apply the change of time $u=s+\gamma(s)$ :

$$
\mathbb{E} \sup _{0 \leq s \leq T}\left|\mathcal{E}_{t}\right|^{2 p} \leq C \mathbb{E}\left[\int_{0}^{\gamma(T)+T}\left(\mathcal{E}_{\tau_{u}}\right)^{2 p} \mathrm{~d} u\right]+C \sqrt{\mathbb{E} \int_{0}^{\gamma(T)+T}\left(\mathcal{E}_{\tau_{u}}\right)^{4 p} \mathrm{~d} u}+C \Delta t^{p} .
$$

By using Lemma 3.4, we have, for $r=1,2$,

$$
\begin{aligned}
\left\{\mathbb{E}\left[\int_{0}^{\gamma(T)+T}\left(\mathcal{E}_{\tau_{u}}\right)^{2 r p} \mathrm{~d} u\right]\right\}^{\frac{1}{r}} & =\left\{\int_{0}^{+\infty} \mathbb{E}\left(\mathbb{1}_{\{\gamma(T)+T \geq u\}}\left(\mathcal{E}_{\tau_{u}}\right)^{2 r p}\right) \mathrm{d} u\right\}^{\frac{1}{r}} \\
& \leq\left\{\int_{0}^{T}\left(\mathcal{E}_{\tau_{u}}\right)^{2 r p} \mathrm{~d} u\right\}^{\frac{1}{r}}+\left\{\int_{T}^{+\infty}[\mathbb{P}(\gamma(T)+T \geq u)]^{\frac{1}{2}}\left[\mathcal{E}_{\tau_{u}}^{4 p r}\right]^{\frac{1}{2}}\right\}^{\frac{1}{r}} \\
& \leq \Delta t^{p}\left[C+C\left\{\int_{0}^{+\infty}[\mathbb{P}(\gamma(T) \geq u)]^{\frac{1}{2}} \exp \left(\frac{u}{2} \mathcal{K}(2 r p)\right) \mathrm{d} u\right\}^{\frac{1}{r}}\right]
\end{aligned}
$$

and finally, we obtain that

$$
\mathbb{E} \sup _{0 \leq s \leq T}\left|\mathcal{E}_{t}\right|^{2 p} \leq \Delta t^{p}\left[C+C \int_{0}^{+\infty}[\mathbb{P}(\gamma(T) \geq u)]^{1 / 2} \exp \left(\frac{u}{2} \mathcal{K}(4 p)\right) \mathrm{d} u\right] .
$$

To finish the proof, we have to show that $u \longrightarrow[\mathbb{P}(\gamma(T) \geq u)]^{1 / 2} \exp \left(\frac{u}{2} \mathcal{K}(4 p)\right) \in L^{1}\left(\mathbb{R}^{+}\right)$. By the Markov inequality, we observe that, for $\mu>0$,

$$
[\mathbb{P}(\gamma(T) \geq u)]^{1 / 2} \leq \exp (-\mu u)(\mathbb{E}[\exp (2 \mu \gamma(T))])^{1 / 2} .
$$


We choose $\mu>\frac{\mathcal{K}(4 p)}{2}$. Furthermore, in view of the definition of $\gamma(T)$ and Lemma 3.1, we have

$$
\mathbb{E}[\exp (2 \mu \gamma(T))] \leq \mathbb{E} \exp \left(2 \mu \int_{0}^{T} \frac{\mathrm{d} s}{X_{s}^{2(1-\alpha)}}\right) \leq C(T, \mu)
$$

Note that in the case $\alpha=1 / 2$, the constraint (10) allows us to choose $2 \mu \leq \frac{\sigma^{2}}{8}\left(\frac{2 b(0)}{\sigma^{2}}-1\right)^{2}$ as required in Lemma 3.1.

\section{REFERENCES}

[1] A. Alfonsi, On the discretization schemes for the CIR (and Bessel squared) processes. Monte Carlo Methods Appl. 11 (2005) 355-384.

[2] A. Berkaoui, Euler scheme for solutions of stochastic differential equations. Potugalia Mathematica Journal 61 (2004) $461-478$.

[3] M. Bossy and A. Diop, Euler scheme for one dimensional SDEs with a diffusion coefficient function of the form $|x|^{a}$, $a$ in $[1 / 2,1)$. Annals Appl. Prob. (Submitted).

[4] M. Bossy, E. Gobet and D. Talay, A symmetrized Euler scheme for an efficient approximation of reflected diffusions. J. Appl. Probab. 41 (2004) 877-889.

[5] J. Cox, J.E. Ingersoll and S.A. Ross, A theory of the term structure of the interest rates. Econometrica 53 (1985) $385-407$.

[6] G. Deelstra and F. Delbaen, Convergence of discretized stochastic (interest rate) processes with stochastic drift term. Appl. Stochastic Models Data Anal. 14 (1998) 77-84.

[7] O. Faure, Simulation du Mouvement Brownien et des Diffusions. Ph.D. thesis, École nationale des ponts et chaussées (1992).

[8] P.S. Hagan, D. Kumar, A.S. Lesniewski and D.E. Woodward, Managing smile risk. WILMOTT Magazine (September, 2002).

[9] J.C. Hull and A. White, Pricing interest-rate derivative securities. Rev. Finan. Stud. 3 (1990) 573-592.

[10] I. Karatzas and S.E. Shreve, Brownian Motion and Stochastic Calculus. Springer-Verlag, New York (1988). 